\title{
Viral dynamics of an HIV model with pulse antiretroviral therapy and adherence
}

\author{
Youping Yang \\ School of Mathematics and Statistics, Shandong Normal University, Jinan, 250014, P. R. China.
}

Communicated by A. Atangana

\begin{abstract}
An immunological model of HIV-1 infection that accounts for antiretroviral drug uptake via explicit compartments is considered. Different from traditional methods where the drug effects is modeled implicitly as a proportional inhibition of viral infection and production, in this paper, it is assumed that the CD4 ${ }^{+} \mathrm{T}$ cells can 'prey on' the antiretroviral drugs and become the cells which cannot be infected or produce new virions. Drug dymamics is modeled applying impulsive differential equations. The basic reproductive number $R_{0}$ is defined via the next infection operator. It is shown that with perfect adherence the virus can be eradicated permanently if $R_{0}$ is less than unity, otherwise, the virus can persist by applying persistent theory. The effects of imperfect adherence are also explored. The results indicate that even for the same degree of adherence, different adherence patterns may lead to different therapy outcomes. In particular, for regular dosage missing, the more dosages are consecutively missed, the worse therapy outcomes will be.
\end{abstract}

Keywords: Impulsive therapy, imperfect adherence, basic reproductive number, adherence pattern.

2010 MSC: 34C60, 37C75, 92B05.

(C)2018 All rights reserved.

\section{Introduction}

Human Immunodeficiency Virus (HIV) is a disease that causes depletion of CD4 ${ }^{+} \mathrm{T}$ cells and can be transmitted by blood or other body fluids [13, 15, 29]. Until recently, there are four classes of antiretroviral (ARV) drugs available for treating HIV-1 infected patients: nucleoside/nucleotide reverse transcriptase inhibitors (NRTIs), non-nucleoside reverse transcriptase inhibitors (NNRTIs), protease inhibitors (PIs), and entry/fusion inhibitors (EIs) [8, 19]. Antiretroviral therapy (ART) containing a combination of three or more drugs chosen from two or more classes has proven to be highly effective in reducing the viral load of HIV infected patients.

There are many mathematical models developed to describe the antiretroviral therapy $[4,8,16,17,27]$ and papers therein. In most of these models, the drug therapy is described by the treatment effect (drug efficacy), which is often assumed to be constant or determined by the plasma drug concentration via

Email address: yyang@sdnu.edu.cn (Youping Yang)

doi: $10.22436 /$ jnsa.011.04.08

Received: 2017-09-18 Revised: 2017-11-17 Accepted: 2018-01-29 
the $E_{\max }$ model $[8,27]$. Our previous work in [30] considered that during drug administration intervals drug concentration varies periodically so as to incorporate the drug dynamics. However, we simplified the model by applying a nonnegative periodic function to represent the drug effects while ignoring the dynamics of drug behavior, which may have a significant impact on certain outcomes [22].

In this paper, we extend the work in [30] by incorporating the drug dynamics into the HIV viral dynamical model via explicit compartments, that is, the $\mathrm{CD} 4^{+} \mathrm{T}$ cells can 'prey on' the antiretroviral drugs, such that $\mathrm{T}$ cells may be susceptible, infected or inhibited by drugs with the drug behavior modeled by impulsive differential equations. This leads to a hybrid system of continuous HIV viral dynamics and discontinuous drug dynamics. Smith and Wahl [23] considered such a model, and gave the long-term dynamics of HIV progression under extreme cases (the drug is administered sufficiently often or the dosage is sufficiently large). However, the authors did not give the full global dynamics of the hybrid system, partially due to the incorporation of the impulses. Hence, our first aim here is to give the threshold parameter (the basic reproductive number) which determines the extinction and the persistence of the virus and investigate the global dynamics of the hybrid system.

Adherence to prescribed ART is recognized as an essential principle in HIV treatment. Imperfect or partial adherence can facilitate the emergence of drug-resistant mutations [6, 15]. In order to determine regimens for partial adherence, a number of mathematical models have been developed to quantify how drug concentration levels in the body of an HIV patient affect viral replication $[5,7-9,12,15,18,21,24,25$, 27]. However, most of these papers analyze single adherence pattern applying classical continuous viral dynamical model $[3,14]$. Based on the importance of understanding the effects of adherence on therapy outcomes, our second aim here is to investigate how different adherence patterns and different degrees of adherence affects the CD4 ${ }^{+} \mathrm{T}$ cell counts and viral load applying the hybrid system.

Our main purpose is then to establish an immunological model of HIV infection incorporating antiretroviral therapy explicitly. We define the basic reproductive number $R_{0}$ applying the next generation operator and study the global dynamics and the persistence of the system. Meanwhile, we study how different degrees of adherence and different adherence patterns will affect the therapy outcomes so as to obtain optimal treatment protocol.

The rest of the paper is organized as follows. In next section, we study the global dynamics of the hybrid system. Then we numerically present how perfect and imperfect adherence (different adherence patterns and different degrees of adherence) affect viral progression in Section 3. We conclude and discuss the results in the final section.

\section{The SIR-type model of PI-sparing therapy}

In this section, we consider an SIR-type model with reverse transcriptase inhibitors or other drugs which prevent cellular infection adapted from [23], and study the global dynamics. Note that we don't consider the loss for the virions during the infection.

$$
\begin{aligned}
\frac{d T_{S}}{d t} & =\lambda-r_{I} T_{S} V_{I}-d_{S} T_{S}-r_{R} T_{S} R+m_{R} T_{R}, \\
\frac{d T_{R}}{d t} & =r_{R} T_{S} R-d_{S} T_{R}-m_{R} T_{R}, \\
\frac{d T_{I}}{d t} & =r_{I} T_{S} V_{I}-d_{I} T_{I}, \\
\frac{d V_{I}}{d t} & =n_{I} \omega T_{I}-d_{V} V_{I} .
\end{aligned}
$$

$\mathrm{R}$ is the plasma drug concentration of the reverse transcriptase inhibitors, satisfying

$$
\frac{d R}{d t}=-d_{R} R, t \neq n \tau, \quad R\left(n \tau^{+}\right)=R(n \tau)+R^{i}, t=n \tau .
$$


We leave out the equation that represents the noninfectious virus $d V_{N I} / d t=n_{I}(1-\omega) T_{I}-d_{V} V_{N I}$, since it can be decoupled from system (2.1).

In the equations, $T_{S}, T_{I}$ denote the susceptible and infected $C D 4^{+} T$ cells, respectively, $T_{R}$ denotes the noninfected $\mathrm{CD}^{+} \mathrm{T}$ cells which have absorbed the reverse transcriptase inhibitors, cells cannot be infected in this state. $V_{I}$ and $V_{N I}$ denote the infectious and non-infectious virus respectively, $n_{I}$ is the number of virions produced per infected cell per day, $\omega$ is the fraction of virions which are infectious produced per day by an infected cell , $d_{V}$ is the rate at which free virus is cleared, $r_{I}$ is the infection rate of free virus, $d_{S}$ and $d_{I}$ are the death rates of noninfected and infected CD4 ${ }^{+} T$ cells, and $r_{R}$ is the rate at which the drug inhibits the T cells. $\lambda$ is the birth rate of $C D 4^{+} \mathrm{T}$ cells, while $m_{R}$ is the rate at which the drug is cleared from the intracellular compartment. Refer to $[15,24]$ for detail descriptions.

We assume that drug is administered with time interval $\tau$, drug dosage $R^{i}$, and the drug is cleared with the rate $d_{R}$. Solving the impulsive differential equations (2.2), we have that the plasma drug concentration ultimately reaches the steady state $R^{*}(t)$ (plateau plasma concentration) $[2,10]$, which is globally asymptomatically stable, and equals

$$
R^{*}(t)=\frac{R^{i}}{1-e^{-d_{R} \tau}} e^{-d_{R}(t-n \tau)}, t \in(n \tau,(n+1) \tau] .
$$

In the following, we will study the hybrid system of (2.1) and (2.2). It is obvious that any solution of system (2.1) with nonnegative initial values is nonnegative. Adding the first three equations of (2.1) we have

$$
\frac{d\left(T_{S}+T_{R}+T_{I}\right)}{d t}=\lambda-d_{S}\left(T_{S}+T_{R}\right)-d_{I} T_{I} \leqslant \lambda-\min \left\{d_{S}, d_{I}\right\}\left(T_{S}+T_{R}+T_{I}\right) .
$$

Hence, $T_{S}+T_{R}+T_{I} \leqslant \lambda / \min \left\{d_{S}, d_{I}\right\}:=\pi_{1}$ as $t \rightarrow \infty$. Then by the fourth equation of (2.1) we have $d V_{I} / d t \leqslant n_{I} \omega \pi_{1}-d_{V} V_{I}$, which follows that $V_{I} \leqslant n_{I} \omega \pi_{1} / d_{V}$ as $t \rightarrow \infty$. Denote $\pi=\max \left\{\pi_{1}, n_{I} \omega \pi_{1} / d_{V}\right\}$, then we have $\left(T_{S}, T_{R}, T_{I}, V_{I}\right) \leqslant(\pi, \pi, \pi, \pi)$ as $t \rightarrow \infty$. We summarize these results in the following theorem.

Theorem 2.1. The solutions of the hybrid system (2.1) and (2.2), with the initial value $\left(\mathrm{T}_{\mathrm{S}_{0}}, \mathrm{~T}_{\mathrm{R} 0}, \mathrm{~T}_{\mathrm{I} 0}, \mathrm{~V}_{\mathrm{IO}}, \mathrm{R}(0)\right) \in$ $\mathbb{R}_{+}^{5}$ are uniformly and ultimately bounded. Furthermore, the compact set $\mathrm{J}=\left\{\left(\mathrm{T}_{\mathrm{S}}, \mathrm{T}_{\mathrm{R}}, \mathrm{T}_{\mathrm{I}}, \mathrm{V}_{\mathrm{I}}\right) \in \mathbb{R}_{+}^{4},\left(\mathrm{~T}_{\mathrm{S}}, \mathrm{T}_{\mathrm{R}}, \mathrm{T}_{\mathrm{I}}, \mathrm{V}_{\mathrm{I}}\right)\right.$ $\leqslant(\pi, \pi, \pi, \pi)\}$ is positively invariant and attracts all positive orbits in $\mathbb{R}_{+}^{4}$.

\subsection{The definition for the basic reproductive number}

In the following, we present the basic reproductive number $R_{0}$ for the hybrid system (2.1) and (2.2) according to the general procedure presented in [1,28] and refer to Yang and Xiao [31].

We start our analysis of systems (2.1) and (2.2) by demonstrating the existence of a periodic 'virus-free' limit cycle. Eqs. (2.1) admit the following solution $T_{I}=V_{I}=0$, with $T_{S}$ and $T_{R}$ dynamics satisfying

$$
\begin{aligned}
& \frac{d T_{S}}{d t}=\lambda-d_{S} T_{S}-r_{R} T_{S} R(t)+m_{R} T_{R}, \\
& \frac{d T_{R}}{d t}=r_{R} T_{S} R(t)-d_{S} T_{R}-m_{R} T_{R} .
\end{aligned}
$$

Adding the two equations of system (2.4), we get

$$
\frac{d\left(T_{S}+T_{R}\right)}{d t}=\lambda-d_{S}\left(T_{S}+T_{R}\right)
$$

Hence, there holds $T_{S}+T_{R} \rightarrow \lambda / d_{S}$, as $t \rightarrow \infty$. Since we consider the long term dynamics of the virus, we substitute $\lambda / d_{S}-T_{S}$ for $T_{R}$, and meanwhile, we apply the plateau plasma drug concentration, then by the first equation we have

$$
\frac{d T_{S}}{d t}=\lambda-d_{S} T_{S}-r_{R} T_{S} R^{*}(t)+m_{R}\left(\frac{\lambda}{d_{S}}-T_{S}\right)=\frac{\lambda}{d_{S}}\left(d_{S}+m_{R}\right)-\left(d_{S}+m_{R}+r_{R} R^{*}(t)\right) T_{S}
$$


System (2.5) admits a globally stable periodic solution which gives

$$
\tilde{T}_{S}(t)=\frac{\lambda}{d_{S}}\left(d_{S}+m_{R}\right) \times \int_{0}^{\infty} \exp \left(-\int_{0}^{s}\left(d_{S}+m_{R}+r_{R} R^{*}(\xi)\right) d \xi\right) d s .
$$

Accordingly, $\tilde{T}_{R}(t)=\lambda / d_{S}-\tilde{T}_{S}(t)$. Hence we get the periodic 'virus-free' solution of system (2.1), that is,

$$
\left(\tilde{T}_{S}(t), \tilde{T}_{R}(t), 0,0\right) \text {. }
$$

Then we define two matrices similar to the next generation matrices.

$$
\mathrm{F}=\left(\begin{array}{cc}
0 & \mathrm{r}_{\mathrm{I}} \tilde{\mathrm{T}}_{\mathrm{S}} \\
0 & 0
\end{array}\right), \quad \mathrm{V}=\left(\begin{array}{cc}
\mathrm{d}_{\mathrm{I}} & 0 \\
-\mathrm{n}_{\mathrm{I}} \omega & \mathrm{d}_{\mathrm{V}}
\end{array}\right)
$$

Let $Y(t, s), t \geqslant s$ be the evolution operator of the linear $\tau$-periodic system

$$
\frac{d y}{d t}=-V y(t)
$$

That is, for each $s \in \mathbb{R}$, the $2 \times 2$ matrix $Y(t, s)$ satisfies

$$
\frac{d Y(t, s)}{d t}=-V Y(t, s), \quad \forall t \geqslant s, Y(s, s)=I,
$$

where $\mathrm{I}$ is a $2 \times 2$ identity matrix.

Define the next infection operator $L$,

$$
(\mathrm{L} \phi)(\mathrm{t})=\int_{-\infty}^{\mathrm{t}} \mathrm{Y}(\mathrm{t}, \mathrm{s}) \mathrm{F}(\mathrm{s}) \phi(\mathrm{s}) \mathrm{ds}, \quad \forall \mathrm{t} \in \mathbb{R}, \phi \in \mathrm{C}_{\omega},
$$

where $C_{\omega}$ is defined as the ordered Banach space of all $\tau$-periodic functions from $\mathbb{R}$ to $\mathbb{R}^{2}$, equipped with the maximum norm $\|\cdot\|$, and the positive cone $C_{\omega}^{+}:=\left\{\phi \in C_{\omega}: \phi(t) \geqslant 0, \forall t \in \mathbb{R}\right\}, \phi(s)$ is the initial distribution of the infected $\mathrm{CD} 4^{+} \mathrm{T}$ cells and the virus.

Note that $F(s) \phi(s)$ is the distribution of the new infections produced by the infected ones introduced at time s. $Y(t, s) F(s) \phi(s), t \geqslant s$ then gives the distribution of the infected individuals who were newly infected at time $s$ and remain infected at time $t$. Then the limit gives the distribution of accumulative new infections at time $t$ which are produced by all those infected individuals $\phi(s)$ introduced at previous time to $t$.

Then according to Wang and Zhao [28], we define the basic reproductive number as the spectral radius of $\mathrm{L}$, that is,

$$
\mathrm{R}_{0}:=\rho(\mathrm{L}) .
$$

In order to characterize $R_{0}$, we consider an auxiliary linear $\tau$-periodic system

$$
\frac{d w}{d t}=\left[-V(t)+\frac{F(t)}{\chi}\right] w, \quad t \in R,
$$

with $\chi \in(0, \infty)$, and $W(t, s, \chi)$ is the evolution operator of system (2.6), $t \geqslant s, s \in \mathbb{R} . \rho(W(\tau, 0, \chi))$ is the spectral radius of the monodromy matrix $W(\tau, 0, \chi)$. We have the following two results, which will be used in our numerical computation of $R_{0}$ and the proof of our main results in Section 2.2.

Lemma 2.2. The following statements are valid.

(i) If $\rho(W(\tau, 0, \chi))=1$ has a positive solution $\chi_{0}$, then $\chi_{0}$ is an eigenvalue of $L$ and hence $R_{0}>0$.

(ii) If $R_{0}>0$, then $\chi=R_{0}$ is the unique solution of $\rho(W(\tau, 0, \chi))=1$.

(iii) $R_{0}=0$ if and only if $\rho(W(\tau, 0, \chi))<1$ for all $\chi>0$. 
Lemma 2.3. The following statements are valid.

(i) $R_{0}=1$ if and only if $\rho\left(\Phi_{\mathrm{F}-\mathrm{V}}(\tau)\right)=1$.

(ii) $R_{0}>1$ if and only if $\rho\left(\Phi_{\mathrm{F}-\mathrm{V}}(\tau)\right)>1$.

(iii) $\mathrm{R}_{0}<1$ if and only if $\rho\left(\Phi_{\mathrm{F}-\mathrm{V}}(\tau)\right)<1$.

The 'virus-free' periodic solution $\left(\tilde{T}_{S}(t), \tilde{T}_{R}(t), 0,0\right)$ is asymptotically stable if $R_{0}<1$, and unstable if $R_{0}>1$.

If the drug concentration $R(t)$ is given by the average plasma drug concentration $R_{a v}$, which is defined as

$$
R_{a v}=\frac{1}{\tau} \int_{0}^{\tau} R^{*}(t) d t=\frac{R^{i}}{\tau d_{R}}
$$

then system (2.1) is an autonomous system. And the 'virus-free' equilibrium equals

$$
\left(T_{S}^{0}, T_{R}^{0}, T_{I}^{0}, V_{I}^{0}\right)=\left(\frac{\lambda}{d_{S}} \times \frac{d_{S}+m_{R}}{d_{S}+m_{R}+r_{R} R_{a v}}, \frac{\lambda}{d_{S}} \times \frac{r_{R} R_{a v}}{d_{S}+m_{R}+r_{R} R_{a v}}, 0,0\right) .
$$

In this case, $F(t) \equiv F, \forall t \geqslant 0$. The use of next generation matrix [26] yields the expression for the basic reproductive number

$$
R_{0}=\rho\left(F V^{-1}\right)=\frac{r_{I} n_{I} \omega}{d_{I} d_{V}} \times \frac{\lambda}{d_{S}} \times \frac{d_{S}+m_{R}}{d_{S}+m_{R}+r_{R} R_{a v}} .
$$

We have the following remark.

Remark 2.4. The basic reproductive number of the time-averaged autonomous system of the periodic system (2.1) will underestimate infection risk.

We illustrate as the drug administration interval varies, how the basic reproductive number of the time-averaged autonomous system, $\left[R_{0}\right]$, given in (2.7) and the basic reproductive number $R_{0}$, calculated applying Lemma 2.2, change. From Figure 1, we can see that the average basic reproductive number $\left[R_{0}\right]$ is always smaller than the basic reproductive number $R_{0}$ when the drug administration interval $\tau$ varies ranging from 0.2 day to 1 day. This implies that the risk of infection will be underestimated if the average basic reproductive number is used.

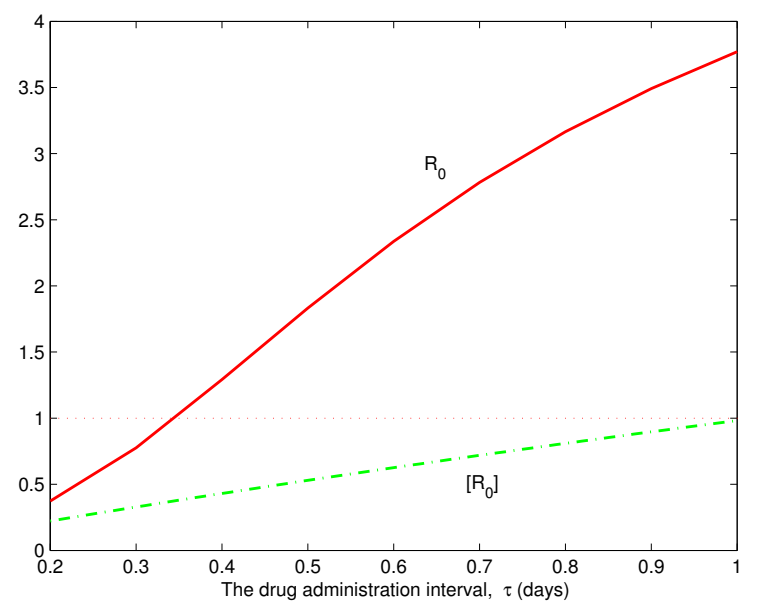

Figure 1: The curves of the basic reproductive number $R_{0}$ and the average basic reproduction number $\left[R_{0}\right]$ versus the drug administration interval $\tau$. Parameters used are $n_{I}=62.5$ day $^{-1}, \omega=0.05, r_{I}=0.0032$ day $^{-1}, d_{S}=0.1$ day $^{-1}, d_{I}=0.5$ day ${ }^{-1}, d_{V}=3$ day $^{-1}, r_{R}=20 \mu M^{-1}, \lambda=100$ cells $\mu L^{-1}, m_{R}=3 \log (2)$ day ${ }^{-1}, R^{i}=7.3 \mu M, d_{R}=16.6$ day $^{-1}$, taken from [23, 24] and references therein.

\subsection{Threshold dynamics}

In this section, we show that the basic reproductive number serves as a threshold parameter that determines the global dynamics. 
Theorem 2.5. If $\mathrm{R}_{0}<1$, then the 'virus-free' periodic solution $\mathrm{P}_{0}=\left(\tilde{\mathrm{T}}_{\mathrm{S}}(\mathrm{t}), \tilde{\mathrm{T}}_{\mathrm{R}}(\mathrm{t}), 0,0\right)$ is globally asymptotically stable.

Proof. The local stability of the 'virus-free' periodic solution $\mathrm{P}_{0}$ when $\mathrm{R}_{0}<1$ is satisfied by Lemma 2.3. Now it is sufficient to prove that $P_{0}$ is globally attractive if $R_{0}<1$.

The plasma drug concentration ultimately reaches the steady state (the plateau drug concentration) $R^{*}(t)$, Eq. (2.3). Then we can choose an integer $n_{1}$ great enough and $t_{1} \geqslant n_{1} \tau$, such that for $t \geqslant t_{1}$, by the first two equations of system (2.1) and the positivity of the solutions for (2.1), we have

$$
\frac{d T_{S}}{d t} \leqslant \lambda-d_{S} T_{S}-r_{R} T_{S} R^{*}+m_{R} T_{R}, \quad \frac{d T_{R}}{d t} \leqslant r_{R} T_{S} R^{*}-d_{S} T_{R}-m_{R} T_{R} .
$$

The corresponding comparison system Eq. (2.4) admits a globally stable periodic solution $\left(\tilde{T}_{S}(t), \tilde{T}_{R}(t)\right)$. Then by the comparison principle, there exists a time $t_{2}>t_{1}$, and $\epsilon>0$, such that $T_{S}(t) \leqslant \tilde{T}_{S}(t)+$ $\epsilon, T_{R}(t) \leqslant \tau_{R}(t)+\epsilon, t \geqslant t_{2}$. By the third and forth equations of system (2.1), for $t \geqslant t_{2}$, we have

$$
\frac{d T_{I}}{d t} \leqslant r_{I}\left(\tilde{T}_{S}(t)+\epsilon\right) V_{I}-d_{I} T_{I}, \quad \frac{d V_{I}}{d t} \leqslant n_{I} \omega T_{I}-d_{V} V_{I} .
$$

Consider an auxiliary system

$$
\frac{d z(t)}{d t}=\left(\begin{array}{cc}
-d_{I} & r_{I} \tilde{T}_{S}(t)+r_{I} \epsilon \\
n_{I} \omega & -d_{V}
\end{array}\right) z(t)=(F(t)-V+\epsilon M) z(t)
$$

where $z=\left(z_{1}, z_{2}\right)^{\top}$, and the matrix

$$
M=\left(\begin{array}{cc}
0 & r_{I} \\
0 & 0
\end{array}\right) .
$$

By Zhang and Zhao [32] (Lemma 2.2), it follows that there exits a positive, $\tau$-periodic function $v(t)$, such that $z(t)=e^{\mu_{1} t} v(t)$ is a solution of system (2.9), where $\mu_{1}=\frac{1}{\tau} \ln \rho\left(\Phi_{(F-V+\epsilon M)}(\tau)\right)$. By Lemma 2.3, we have $\rho\left(\Phi_{F-V}(\tau)\right)<1$. Since $\rho\left(\Phi_{(F-V+\epsilon M)}(\tau)\right)$ is continuous for small $\epsilon$, we can choose $\epsilon$ small enough such that $\rho\left(\Phi_{(F-V+\epsilon M)}(\tau)\right)<1$, that is $\mu_{1}<0$. Therefore, we have $z(t) \rightarrow 0$, as $t \rightarrow \infty$. For any nonnegative initial value $\left(\mathrm{T}_{\mathrm{I}}\left(\mathrm{t}_{2}\right), \mathrm{V}_{\mathrm{I}}\left(\mathrm{t}_{2}\right)\right)^{\mathrm{T}}$ of system (2.8), there exits a $z^{*}$ large enough such that $\left(\mathrm{T}_{\mathrm{I}}\left(\mathrm{t}_{2}\right), \mathrm{V}_{\mathrm{I}}\left(\mathrm{t}_{2}\right)\right)^{\mathrm{T}} \leqslant z^{*}\left(v_{1}(0), v_{2}(0)\right)^{\mathrm{T}}$. Then the comparison theorem indicates that

$$
\left(T_{I}(t), V_{I}(t)\right)^{\top} \leqslant z^{*} e^{\mu_{1}\left(t-t_{2}\right)}\left(v_{1}\left(t-t_{2}\right), v_{2}\left(t-t_{2}\right)\right)^{\top}, \quad \forall t \geqslant t_{2}
$$

Hence, we have $T_{I}(t) \rightarrow 0, V_{I}(t) \rightarrow 0$ as $t \rightarrow \infty$. By the fist and second equations of system (2.1), we have $\mathrm{T}_{S}(\mathrm{t}) \rightarrow \tilde{\mathrm{T}}_{S}(\mathrm{t}), \mathrm{T}_{\mathrm{R}}(\mathrm{t}) \rightarrow \tilde{\mathrm{T}}_{\mathrm{R}}(\mathrm{t})$ as $\mathrm{t} \rightarrow \infty$. We get the global asymptotical stability of the 'virus-free' periodic solution. This completes the proof.

In order to better understand the proof of the following theorem for the readers, we present some notations and a lemma first.

Define

$$
X=R_{+}^{4}, \quad X_{0}=\left\{\left(T_{S}, T_{R}, T_{I}, V_{I}\right) \in X: T_{I}>0, V_{I}>0\right\}, \quad \partial X_{0}:=X \backslash X_{0}
$$

Let $P: X \rightarrow X$ be Poincaré map associated with system (2.1), satisfying $P\left(x^{0}\right)=u\left(\tau, x^{0}\right), \forall x^{0} \in X, u\left(t, x^{0}\right)$ is the unique solution of $(2.1)$ with $u\left(0, x^{0}\right)=x^{0}$. It can be seen easily that

$$
\mathrm{P}^{\mathrm{m}}\left(\mathrm{T}_{\mathrm{S} 0}, \mathrm{~T}_{\mathrm{R} 0}, \mathrm{~T}_{\mathrm{I} 0}, \mathrm{~V}_{\mathrm{I} 0}\right)=\mathrm{u}\left(\mathrm{m} \tau,\left(\mathrm{T}_{\mathrm{S} 0}, \mathrm{~T}_{\mathrm{R} 0}, \mathrm{~T}_{\mathrm{I} 0}, \mathrm{~V}_{\mathrm{I} 0}\right)\right), \forall \mathrm{m} \geqslant 0 .
$$

Clearly, the fixed point of the Poincare map $P$ in $X$ is $M_{1}=\left(\tilde{T}_{S}(0), \tilde{T}_{R}(0), 0,0\right)$.

Lemma 2.6. If the basic reproductive number $\mathrm{R}_{0}>1$, then there exists a $\delta_{0}>0$ such that for all $\chi^{0} \in \mathrm{X}_{0}$ with $\left\|x^{0}-M_{1}\right\| \leqslant \delta_{0}$, where $x^{0}=\left(T_{S 0}, T_{R 0}, T_{I 0}, V_{I 0}\right) \in X_{0}$, there holds

$$
\limsup _{m \rightarrow \infty} d\left(P^{m}\left(x^{0}\right), M_{1}\right) \geqslant \delta_{0} \text {. }
$$


Proof. By the continuity of solutions with respect to the initial values, $\forall \epsilon>0$ there exists $\delta_{0}>0$ such that for all $x^{0} \in X_{0}$ with $\left\|x^{0}-M_{1}\right\| \leqslant \delta_{0}$, there holds $\left.\| u\left(t, x^{0}\right)-u\left(t, M_{1}\right)\right) \| \leqslant \epsilon, \forall t \in[0, w]$. We further claim that Eq. (2.10) holds. Assume, by contradiction, that (2.10) does not hold. Then we have

$$
\limsup _{m \rightarrow \infty} d\left(P^{m}\left(x^{0}\right), M_{1}\right)<\delta_{0}
$$

for some $x^{0} \in X_{0}$. Without loss of generality, we assume that $d\left(P^{m}\left(x^{0}\right), M_{1}\right)<\delta_{0}$ for all $m \geqslant 0$. It follows that

$$
\left\|u\left(t, P^{m}\left(x^{0}\right)\right)-u\left(t, M_{1}\right)\right\| \leqslant \epsilon, \forall t \in[0, w] .
$$

For any $t \geqslant 0$, let $t=m \omega+t^{\prime}$, where $t^{\prime} \in[0, \omega]$ and $m=\left[\frac{t}{\omega}\right]$ is the greatest integer less than or equal to $\frac{t}{\omega}$. Thus, we get

$$
\left\|u\left(t, x^{0}\right)-u\left(t, M_{1}\right)\right\|=\left\|u\left(t^{\prime}, P^{m}\left(x^{0}\right)\right)-u\left(t^{\prime}, M_{1}\right)\right\|<\epsilon, \forall t \geqslant 0 .
$$

Note that $\left(T_{S}(t), T_{R}(t), T_{I}(t), V_{I}(t)\right)=u\left(t, x^{0}\right)$. It then follows that $T_{I}(t)<\epsilon, V_{I}(t)<\epsilon, \forall t \geqslant 0$. Then from the first and second equations of (2.1), we have

$$
\frac{d T_{S}}{d t} \geqslant \lambda-d_{S} T_{S}-\epsilon r_{I} T_{S}-r_{R} T_{R} R+m_{R} T_{R}, \quad \frac{d T_{R}}{d t} \geqslant r_{R} T_{R} R-d_{S} T_{R}-m_{R} T_{R}
$$

Consider an auxiliary equation

$$
\frac{d \hat{T}_{S}}{d t}=\lambda-d_{S} \hat{T}_{S}-\epsilon r_{I} \hat{T}_{S}-r_{R} \hat{T}_{R} R+m_{R} \hat{T}_{R}, \quad \frac{d \hat{T}_{R}}{d t}=r_{R} \hat{T}_{R} R-d_{S} \hat{T}_{R}-m_{R} \hat{T}_{R}
$$

For any $\epsilon>0$, system (2.11) admits a globally attractive solution $\left(\hat{T}_{S}(0, \epsilon), \hat{T}_{R}(0, \epsilon)\right)$. Then for any $\xi>0$, there exists $t_{3}>0$ such that $\hat{T}_{S}(t, \epsilon) \geqslant \hat{T}_{S}(0, \epsilon)-\xi$, and $\hat{T}_{R}(t, \epsilon) \geqslant \hat{T}_{R}(0, \epsilon)-\xi$ for $t \geqslant t_{3},\left(\hat{T}_{S}(t, \epsilon), \hat{T}_{R}(t, \epsilon)\right)$ is any solution of (2.11). Note that $\hat{T}_{S}(0, \epsilon) \rightarrow \tilde{T}_{S}(t), \hat{T}_{R}(0, \epsilon) \rightarrow \tilde{T}_{R}(t)$ as $\epsilon \rightarrow 0$. Then for any $\bar{\eta}>0$ there exists $\bar{\epsilon}>0$ such that $\hat{T}_{S}(0, \epsilon) \geqslant \tilde{T}_{S}(t)-\bar{\eta}, \hat{T}_{R}(0, \epsilon) \geqslant \tilde{T}_{R}(t)-\bar{\eta}$ for $\epsilon<\bar{\epsilon}$. It follows that for $t \geqslant t_{3}$ and $\epsilon$ small enough $(\epsilon<\bar{\epsilon})$

$$
\begin{aligned}
& \hat{\mathrm{T}}_{S}(t, \epsilon) \geqslant \hat{\mathrm{T}}_{S}(0, \epsilon)-\xi \geqslant \tilde{\mathrm{T}}_{S}(\mathrm{t})-\bar{\eta}-\xi \triangleq \tilde{\mathrm{T}}_{S}(\mathrm{t})-\eta, \\
& \hat{\mathrm{T}}_{\mathrm{R}}(\mathrm{t}, \epsilon) \geqslant \hat{\mathrm{T}}_{\mathrm{R}}(0, \epsilon)-\xi \geqslant \tilde{\mathrm{T}}_{\mathrm{R}}(\mathrm{t})-\bar{\eta}-\xi \triangleq \tilde{\mathrm{T}}_{\mathrm{R}}(\mathrm{t})-\eta .
\end{aligned}
$$

Then the comparison principle indicates that $T_{S}(t) \geqslant \hat{T}_{S}(t, \epsilon) \geqslant \tilde{T}_{S}(t)-\eta$ for $t \geqslant t_{3}$ and $\epsilon$ small enough.

Consider another auxiliary system

$$
\left(\begin{array}{c}
\frac{d \breve{T}_{I}(t)}{d t} \\
\frac{d \breve{V}_{I}(t)}{d t}
\end{array}\right)=\left(\begin{array}{cc}
-d_{I} & r_{I} \tilde{T}_{S}(t)-\eta r_{I} \\
n_{I} \omega & -d_{V}
\end{array}\right)\left(\begin{array}{c}
\breve{T}_{I}(t) \\
\breve{V}_{I}(t)
\end{array}\right)=(F(t)-V-\eta M)\left(\begin{array}{c}
\breve{T}_{I}(t) \\
\breve{V}_{I}(t)
\end{array}\right) .
$$

By Zhang and Zhao [32] (Lemma 2.2), we know that there exists a positive, $\tau$-periodic function $p(t)=$ $\left(p_{1}(t), p_{2}(t)\right)$, such that $e^{\mu_{2} t} p(t)$ is a solution of system (2.12), where $\mu_{2}=\frac{1}{\tau} \ln \rho\left(\Phi_{F-V-\eta M}(\tau)\right)$. Since $\rho\left(\Phi_{F-V-\eta M}(\tau)\right)$ is continuous for small $\eta$ and $\rho\left(\Phi_{F-V}(\tau)\right)>1$, we can choose $\eta$ small enough such that $\rho\left(\Phi_{\mathrm{F}-V-\eta M}(\tau)\right)>1$, that is $\mu_{2}>0$. Let $t=n \tau>t_{3}$, and $n$ be nonnegative integer, we get

$$
\left(\breve{T}_{I}(n \tau), \breve{V}_{I}(n \tau)\right)^{\top}=e^{\mu_{2}\left(n \tau-t_{3}\right)}\left(p_{1}\left(n \tau-t_{3}\right), p_{2}\left(n \tau-t_{3}\right)\right)^{\top} \rightarrow(\infty, \infty)^{\top}, n \rightarrow \infty .
$$

For any negative initial values $\left(T_{I}\left(t_{3}\right), V_{I}\left(t_{3}\right)\right)^{T}$ of the comparison system of (2.12), there exits a sufficiently small $z_{*}>0$, such that $\left(\mathrm{T}_{\mathrm{I}}\left(\mathrm{t}_{3}\right), \mathrm{V}_{\mathrm{I}}\left(\mathrm{t}_{3}\right)\right)^{\mathrm{T}} \geqslant z_{*}\left(\mathrm{p}_{1}(0), \mathrm{p}_{2}(0)\right)^{\mathrm{T}}$. By the comparison theorem, we have $\left(T_{I}(t), V_{I}(t)\right)^{T} \geqslant z_{*} e^{\mu_{2}\left(t-t_{3}\right)}\left(\breve{T}_{I}\left(t-t_{3}\right), \breve{V}_{I}\left(t-t_{3}\right)\right)^{T}$ for all $t \geqslant t_{3}$. Thus we obtain $T_{I}(n \tau) \rightarrow \infty, V_{I}(n \tau) \rightarrow$ $\infty$, as $n \rightarrow \infty$, a contradiction. Hence Eq. (2.10) holds. This completes the proof.

Theorem 2.7. If the basic reproductive number $\mathrm{R}_{0}>1$, then there exists a $\delta>0$ such that any solution 
$\left(T_{S}(t), T_{R}(t), T_{I}(t), V_{I}(t)\right)$ of system (2.1) with initial value $\left(T_{S 0}, T_{R 0}, T_{I 0}, V_{I 0}\right) \in\left\{\left(T_{S}, T_{R}, T_{I}, V_{I}\right) \in X \mid T_{I}>\right.$ $\left.0, V_{I}>0\right\}$ satisfies

$$
\liminf _{t \rightarrow \infty} T_{I}(t) \geqslant \delta, \quad \liminf _{t \rightarrow \infty} V_{I}(t) \geqslant \delta,
$$

and system (2.1) admits at least one positive periodic solution.

Proof. In the following we will show that $\mathrm{P}$ is uniformly persistent with respect to $\left(\mathrm{X}_{0}, \partial \mathrm{X}_{0}\right)$. Due to the uniform and ultimate boundedness of the solutions of system (2.1), the Poincaré map $P$ admits a global attractor in $X$. For any $\left(T_{S 0}, T_{R 0}, T_{I 0}, V_{I 0}\right) \in X_{0}$, the first equation of system (2.1) can be written as

$$
\frac{d T_{S}}{d t} \geqslant \lambda-d_{S} T_{S}-r_{I} V_{I} T_{S}-r_{R} R T_{S}
$$

Then we have

$$
\begin{aligned}
& \mathrm{T}_{\mathrm{S}}(\mathrm{t}) \geqslant \exp \left(-\int_{0}^{\mathrm{t}}\left(\mathrm{d}_{\mathrm{S}}+\mathrm{r}_{\mathrm{I}} \mathrm{V}_{\mathrm{I}}\left(\mathrm{s}_{1}\right)+\mathrm{r}_{\mathrm{R}} \mathrm{R}\left(\mathrm{s}_{1}\right)\right) \mathrm{d} \mathrm{s}_{1}\right) \\
& \times\left(\mathrm{T}_{\mathrm{S}}(0)+\lambda \int_{0}^{\mathrm{t}} \exp \left(\int_{0}^{\sigma}\left(\mathrm{d}_{\mathrm{S}}+\mathrm{r}_{\mathrm{I}} \mathrm{V}_{\mathrm{I}}\left(\mathrm{s}_{2}\right)+\mathrm{r}_{\mathrm{R}} \mathrm{R}\left(\mathrm{s}_{2}\right)\right) \mathrm{d} \mathrm{s}_{2}\right) \mathrm{d} \sigma\right) \\
& \geqslant \exp \left(-\int_{0}^{t}\left(d_{S}+r_{I} V_{I}\left(s_{1}\right)+r_{R} R\left(s_{1}\right)\right) d s_{1}\right) \\
& \times \lambda \int_{0}^{t} \exp \left(\int_{0}^{\sigma}\left(d_{S}+r_{I} V_{I}\left(s_{2}\right)+r_{R} R\left(s_{2}\right)\right) d s_{2}\right) d \sigma, \\
& >0, \quad \forall \mathrm{t}>0 \text {. }
\end{aligned}
$$

By the second equation of system (2.1) we can deduce that $T_{R}(t)>0$ for all $t>0$. Furthermore, by Theorem 4.1.1 in [20] as generalized to nonautonomous systems, the irreducibility of the cooperative matrix

$$
\left(\begin{array}{cc}
-d_{I} & r_{I} T_{S}(t) \\
n_{I} \omega & -d_{V}
\end{array}\right)
$$

implies that $T_{I}(t)>0, V_{I}(t)>0$ for all $t>0$. Thus, both $X$ and $X_{0}$ are positively invariant. Clearly, $\partial X_{0}$ is relatively closed in $X$.

Set

$$
M_{\partial}=\left\{\left(T_{S 0}, T_{R 0}, T_{I 0}, V_{I 0}\right) \in \partial X_{0}: P^{m}\left(T_{S 0}, T_{R 0}, T_{I 0}, V_{I 0}\right) \in \partial X_{0}, \forall m \geqslant 0\right\}
$$

We now show that

$$
M_{\partial}=\left\{\left(T_{S}, T_{R}, 0,0\right): T_{S} \geqslant 0, T_{R} \geqslant 0\right\} .
$$

It suffices to prove that for any $\left(T_{S 0}, T_{R 0}, T_{I 0}, V_{I 0}\right) \in M_{\partial}$, there holds $T_{I}(m \tau)=V_{I}(m \tau)=0, \forall m \geqslant 0$. Suppose not, then there exists an $m_{1} \geqslant 0$ such that $T_{I}\left(m_{1} \tau\right)>0, V_{I}\left(m_{1} \tau\right)>0$. Set the initial time in Eq. (2.13) to be $m_{1} \tau$, then $T_{S}(t)>0, \forall t>m_{1} \tau$. Applying similar method, we have $T_{I}(t)>0, V_{I}(t)>0, \forall t>$ $m_{1} \tau$, with the initial value $T_{I}\left(m_{1} \tau\right)>0, V_{I}\left(m_{1} \tau\right)>0$. Thus we have

$$
\left(T_{S}(t), T_{R}(t), T_{I}(t), V_{I}(t)\right) \in X_{0}, \forall t>m_{1} \tau,
$$

which contradicts with the assumption that $\left(T_{S 0}, T_{R 0}, T_{I 0}, V_{I 0}\right) \in M_{\partial}$, hence (2.14) holds. Clearly, there is exactly one fixed point of $P$ in $M_{\partial}$, which is $M_{1}=\left(\tilde{T}_{S}(0), \tilde{T}_{R}(0), 0,0\right)$.

Lemma 2.6 implies that $M_{1}$ is isolated in $X$ and $W^{s}\left(M_{1}\right) \cap X_{0}=\emptyset$. Clearly, each orbit in $M_{\partial}$ converges to $M_{1}$, and $M_{1}$ is acyclic in $M_{\partial}$. By Theorem 1.3.1 in [33], it follows that $P$ is uniformly persistent with respect to $\left(X_{0}, \partial X_{0}\right)$. By Theorem 3.1.1 in [33] the solutions of (2.1) are uniformly persistent.

Furthermore, Theorem 1.3.6 in [33] implies that $P$ has a fixed point $\left(\mathrm{T}_{S}^{*}(0), \mathrm{T}_{\mathrm{R}}^{*}(0), \mathrm{T}_{\mathrm{I}}^{*}(0), \mathrm{V}_{\mathrm{I}}^{*}(0)\right) \in X_{0}$. Then $\mathrm{T}_{S}^{*}(0) \geqslant 0, \mathrm{~T}_{\mathrm{R}}^{*}(0) \geqslant 0, \mathrm{~T}_{\mathrm{I}}^{*}(0)>0, \mathrm{~V}_{\mathrm{I}}^{*}(0)>0$. We further claim that there exists $\overline{\mathrm{t}} \in[0, \omega]$, such that $\mathrm{T}_{\mathrm{S}}^{*}(\overline{\mathrm{t}})>0, \mathrm{~T}_{\mathrm{R}}^{*}(\overline{\mathrm{t}})>0$. Suppose not, by the periodicity of $\mathrm{T}_{\mathrm{S}}^{*}(\mathrm{t})$ and $\mathrm{T}_{\mathrm{R}}^{*}(\mathrm{t})$, there holds $\mathrm{T}_{\mathrm{S}}^{*}(\mathrm{t}) \equiv 0, \mathrm{~T}_{\mathrm{R}}^{*}(\mathrm{t}) \equiv 0$ 
for all $t \geqslant 0$. Then adding the first two equations of (2.1), we have $0<\lambda=0$, a contradiction. The first equation of system (2.1) can be written as

$$
\frac{d T_{S}}{d t} \geqslant \lambda-d_{S} T_{S}-r_{I} V_{I} T_{S}-r_{R} R T_{S}
$$

Then we have

$$
\begin{aligned}
\mathrm{T}_{\mathrm{S}}^{*}(\mathrm{t}) \geqslant & \exp \left(-\int_{\overline{\mathrm{t}}}^{\mathrm{t}}\left(\mathrm{d}_{\mathrm{S}}+\mathrm{r}_{\mathrm{I}} \mathrm{V}_{\mathrm{I}}\left(\mathrm{s}_{1}\right)+\mathrm{r}_{\mathrm{R}} \mathrm{R}\left(\mathrm{s}_{1}\right)\right) \mathrm{d} \mathrm{s}_{1}\right) \\
& \times\left(\mathrm{T}_{\mathrm{S}}^{*}(\overline{\mathrm{t}})+\lambda \int_{\overline{\mathrm{t}}}^{\mathrm{t}} \exp \left(\int_{\overline{\mathrm{t}}}^{\sigma}\left(\mathrm{d}_{\mathrm{S}}+\mathrm{r}_{\mathrm{I}} \mathrm{V}_{\mathrm{I}}\left(\mathrm{s}_{2}\right)+\mathrm{r}_{\mathrm{R}} \mathrm{R}\left(\mathrm{s}_{2}\right)\right) d \mathrm{~s}_{2}\right) d \sigma\right) \\
> & 0, \quad \forall \mathrm{t} \in[\overline{\mathrm{t}}, \overline{\mathrm{t}}+\omega] .
\end{aligned}
$$

Thus $T_{S}^{*}(t)>0$ for all $t \geqslant 0$ by the periodicity. By the second equation of system (2.1) we can deduce that $\mathrm{T}_{\mathrm{R}}^{*}(\mathrm{t})>0$ for all $\mathrm{t} \geqslant 0$. Furthermore, by Theorem 4.1.1 in [20] as generalized to nonautonomous systems, the irreducibility of the cooperative matrix [11]

$$
\left(\begin{array}{cc}
-d_{I} & r_{I} T_{S}^{*}(t) \\
n_{I} \omega & -d_{V}
\end{array}\right)
$$

implies that $T_{I}^{*}(t)>0, V_{I}^{*}(t)>0$ for all $t \geqslant 0$. Therefore $\left(T_{S}^{*}(t), T_{R}^{*}(t), T_{I}^{*}(t), V_{I}^{*}(t)\right)$ is a positive periodic solution of system (2.1). This completes the proof.

\section{Numerical simulations}

We consider two therapy strategies.

1. Perfect adherence: drugs are administered with time interval $\tau$ and dosage $R^{i}$.

2. Imperfect adherence: drugs are also administered with time interval $\tau$, except for a finite time halted when some dosages are missed.

The parameters used are as those in Figure 1. First for perfect adherence, we have shown theoretically that if the basic reproductive number $\mathrm{R}_{0}<1$, the 'virus-free' periodic solution is globally asymptomatically stable, while if $R_{0}>1$, at least a positive periodic solution exists. Fix the administration interval $\tau=0.3$ day, by calculation $R_{0}=0.776<1$, when $\tau=0.5$ day, $R_{0}=1.8321>1$. The simulation results in Figures 2 and 3 verify Theorems 2.5 and 2.7 and show that the positive periodic solution is also globally attractive.
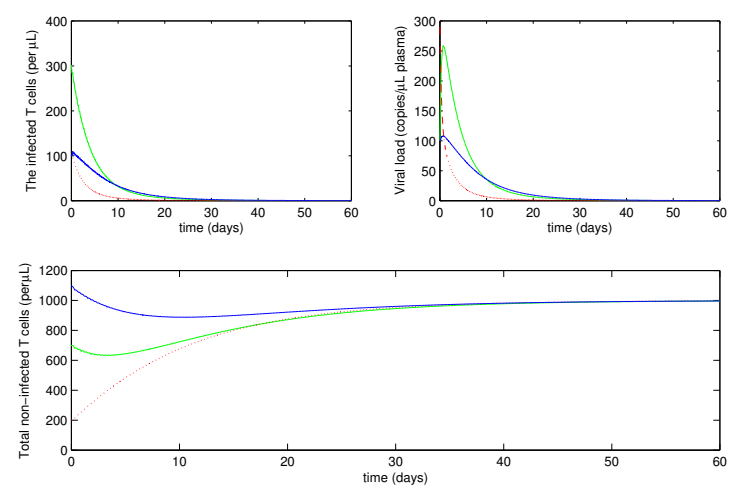

Figure 2: The global asymptomatical stability of the 'virus-free' periodic solution when the basic reproductive number $\mathrm{R}_{0}=$ $0.776<1, \tau=0.3$ day. 

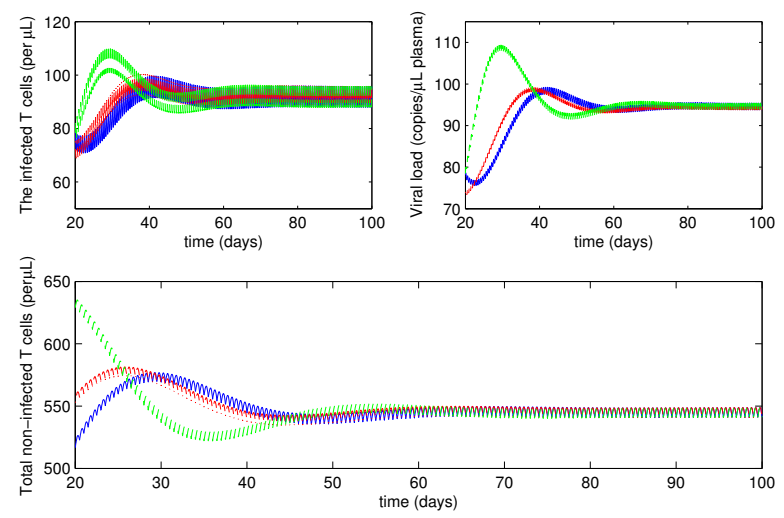

Figure 3: The global asymptomatical stability of the positive periodic solution when the basic reproductive number $R_{0}=1.8321>$ $1, \tau=0.5$ day.

To model imperfect adherence, let $p$ denote the fraction of the prescribed doses of the drug which are taken. Now a natural question arises: given the same degree of adherence, how will different adherence patterns affect viral dynamics and long-term prediction of therapy? We attempt to address this question in the following.
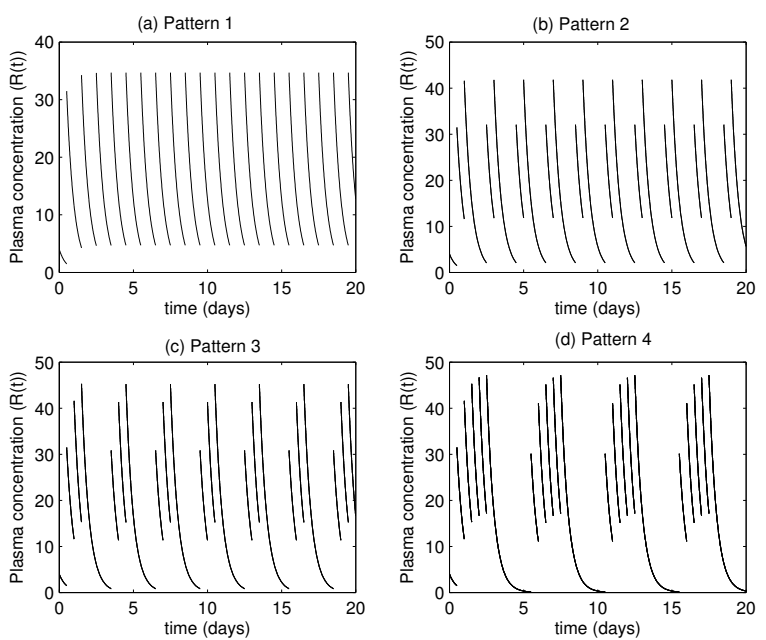

Figure 4: Plasma concentration time curves with the adherence $p=0.5$.

In Figure 4, we present four adherence patterns with the same value of $p=0.5$. Figure 4 (a) shows the plasma drug concentration when every other dose is missed (Pattern 1). Pattern 2 shows two doses are taken followed by two doses missed. Pattern 3 shows three doses are taken followed by three doses missed and Pattern 4 shows five doses are taken followed by five doses missed. Patterns 1-4 describe different adherence patterns by the block size, which is defined as the number of consecutive doses taken or missed each time a dose is taken or missed [8,27].

We present in Figure 5 the time evolution of the total non-infected T cells, the infected T cells, and the viral load for Pattern 1 to Pattern 4 with adherence $p=0.5$. With perfect adherence, the virus will be eliminated from the host, see from Figure 2. For imperfect adherence, from Figure 5 we can see clearly that Pattern 1 performs better than the other patterns with three or five consecutive missed doses in keeping the total non-infected $\mathrm{T}$ cells at a higher level, while keeping the infected $\mathrm{T}$ cells and the viral load at relatively lower levels. This indicates that smaller block size performs better in reducing viral load. 

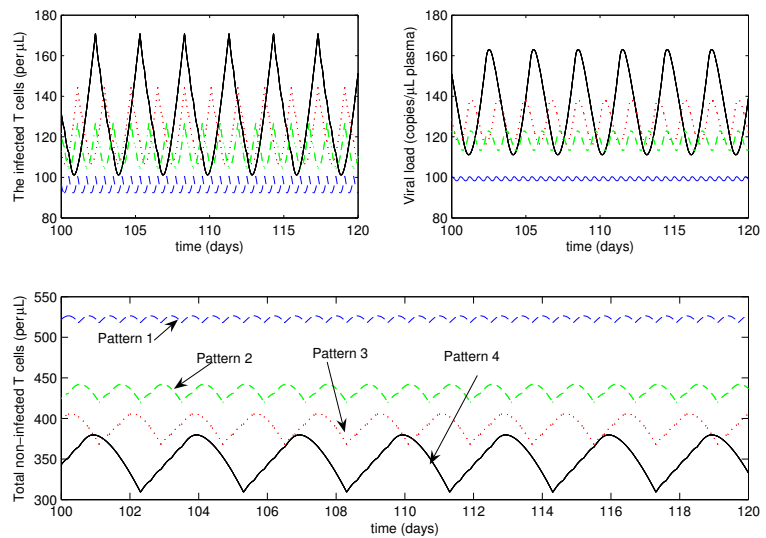

Figure 5: A comparison of the immunologic and viral responses based on the four patterns of adherence with $p=0.5$ displayed in Figure 4.

The adherence patterns we have considered are regular. Note that for the same degree of adherence $p=0.5$, there are other adherence patterns, some of which may be irregular. We do not consider these irregular patterns, for they will lead to non-periodic systems such that the analytical results cannot be applied. Further studies may be focused on some numerical results.

When the degree of adherence $p$ varies, numerical simulations indicate that with regular drug dosages 'taken and missed', as the adherence $p$ increases, drug therapy performs better in reducing viral load at lower levels. For example, drugs that are taken five doses followed by five consecutive missed doses (according to $p=1 / 2$ ) perform better than drug that is taken one dose followed by two doses missed (according to $p=1 / 3$ ) (figures are not shown).

\section{Conclusions and discussions}

In this paper, we consider an immunological model for HIV viral dynamics incorporating the effects of the reverse transcriptase inhibitors explicitly. There are many authors considering HIV drug therapy implicitly $[4,8,16,17,27]$. In those classical models, the effects of the drugs are assumed to reduce the infection rate (PI sparing therapy) by a fraction $1-n_{r t}$, where $n_{r t}$ is assumed to be the drug efficacy. The basic reproductive number in this case is then

$$
R_{0}=\frac{\left(1-n_{r t}\right) r_{I} n_{I} \omega}{d_{I} d_{V}} \times \frac{\lambda}{d_{S}}
$$

If the drug is explicitly modeled as that in system (2.1), we consider the case when the drug varies with sufficiently small amplitude, that is the drug concentration can be seen as a constant, i.e., $R(t) \equiv R, \forall t \geqslant 0$. Then the basic reproductive number yields

$$
R_{0}=\frac{r_{I} n_{I} \omega}{d_{I} d_{V}} \times \frac{\lambda}{d_{S}} \times \frac{d_{S}+m_{R}}{d_{S}+m_{R}+r_{R} R} .
$$

From the expressions for the basic reproductive numbers we can see that when the drug is implicitly modeled, it acts to reduce the infection rate $r_{I}$, while if the drug is explicitly modeled, that is, the $T$ cells may be susceptible, infected or inhibited by the drug, in this case since the uninfected $\mathrm{T}_{\text {cells }} \mathrm{T}_{\mathrm{S}}$ will absorb the drug and become cells $T_{R}$ which cannot be infected further, then the susceptible $T$ cells will be reduced by a fraction $\left(d_{S}+m_{R}\right) /\left(d_{S}+m_{R}+r_{R} R\right)$. Hence, the implicit model and the explicit model act in different ways to reduce the number of $T$ cells that will be infected by free virus.

Our main results show that for perfect adherence, the virus-free periodic solution of system (2.1) is globally asymptotically stable if $R_{0}<1$, whilst the virus is persistent if $R_{0}>1$, and meanwhile there 
exists a positive periodic solution, which is globally asymptotically stable numerically. Since the basic reproductive number $R_{0}$ is the function of the drug dosage $R^{i}$ and the drug administration interval $\tau$, hence we can design the regimen strategy to ensure the elimination of the virus theoretically.

For imperfect adherence, different degrees of adherence and different adherence patterns will affect therapy outcomes differently. Given the same degree, smaller blocking size performs better in reducing viral load. With regular dosages taken and missed, the bigger the degree of the adherence, the better the therapy outcome will be.

\section{Acknowledgment}

This work is supported by the National Natural Science Foundation of China (NSFC 11401349) and the Foundation for Outstanding Young Scientist in Shandong Province (BS2014SF008).

\section{References}

[1] N. Bacaër, S. Guernaoui, The epidemic threshold of vector-borne diseases with seasonality, Morocco. J. Math. Biol., 53 (2006), 421-436. 2.1

[2] D. Baĭnov, P. Simeonov, Impulsive differential equations: periodic solutions and applications, Pitman Monographs and Surveys in Pure and Applied Mathematics, Longman Scientific \& Technical, Harlow; copublished in the United States with John Wiley \& Sons, Inc., New York, (1993). 2

[3] S. Bonhoeffer, M. A. Nowak, Pre-existence and emergence of drug resistance in HIV-1 infection, Proc. R. Soc. Lond. B, 264 (1997), 631-637. 1

[4] A. A. Ding, H. Wu, Assessing antiviral potency of anti-HIV therapies in vivo by comparing viral decay rates in viral dynamic models, Biostatistics, 2 (2001), 13-29. 1, 4

[5] N. M. Ferguson, C. A. Donnelly, J. Hooper, A. C. Ghani, C. Fraser, L. M. Bartley, R. A. Rode, P. Vernazza, D. Lapins, S. L. Mayer, R. M. Anderson, Adherence to antiretroviral therapy and its impact on clinical outcome in HIVinfected patients, J. R. Soc. Interface., 2 (2005), 349-363. 1

[6] G. H. Friedland, A. Williams, Attaining higher goals in HIV treatment: the central importance of adherence, AIDS, 13 (1999), 61-72. 1

[7] Y. Huang, D. Liu, H. Wu, Hierarchical Bayesian methods for estimation of parameters in longitudinal HIV dynamic system, Biometrics, 62 (2006), 413-423. 1

[8] Y. Huang, S. L. Rosenkranz, H. Wu, Modeling HIV dynamics and antiviral response with consideration of time-varying drug exposures, adherence and phenotypic sensitivity, Math. Biosci., 184 (2003), 165-186. 1, 3, 4

[9] O. Krakovska, L. M. Wahl, Optimal drug treatment regimens for HIV depend on adherence, J. Theoret. Biol., 246 (2007), 499-509. 1

[10] V. Lakshmikantham, D. D. Baŭnov, P. S. Simeonov, Theory of impulsive differential equations, World Scientific Publishing Co., Inc., Teaneck, New Jersey, (1989). 2

[11] L. Liu, X.-Q. Zhao, Y. Zhou, A Tuberculosis Model with Seasonality, Bull. Math. Biol., 72 (2010), 931-952. 2.2

[12] J. Lou, R. J. Smith, Modelling the effects of adherence to the HIV fusion inhibitor enfuvirtide, J. Theoret. Biol., 268 (2011), 1-13. 1

[13] J. M. McCune, The dynamics of $\mathrm{CD}^{+}{ }^{+}$T-cell depletion in HIV disease, Nature, 410 (2001), 974-979. 1

[14] A. R. McLean, M. A. Nowak, Competition between zidovudine-sensitive and zidovudine-resistant strains of HIV, AIDS, 6 (1992), 71-79. 1

[15] R. E. Miron, R. J. Smith, Modelling imperfect adherence to HIV induction therapy, BMC Infect. Dis., 2010 (2010), 16 pages. 1,2

[16] M. A. Nowak, S. Bonhoeffer, G. M. Shaw, R. M. May, Anti-viral drug treatment: dynamics of resistance in free virus and infected cell populations, J. Theor. Biol., 184 (1997), 203-217. 1, 4

[17] A. S. Perelson, P. W. Nelson, Mathematical analysis of HIV-1 dynamics in vivo, SIAM Rev., 41 (1999), 3-44. 1, 4

[18] A. N. Philips, M. Youlem, M. Johnson, C. Loveday, Use of stochastic model to develop understanding of the impact of different patterns of antiretroviral drug use on resistance development, AIDS, 15 (2001), 2211-2220. 1

[19] L. Rong, Z. Feng, A. S. Perelson, Emergence of HIV-1 Drug Resistance During Antiretroviral Treatment, Bull. Math. Biol., 69 (2007), 2027-2060. 1

[20] H. L. Smith, Monotone Dynamical Systems: An Introduction to the Theory of Competitive and Cooperative Systems, Mathematical Surveys and Monographs, American Mathematical Society, Providence, (1995). 2.2, 2.2

[21] R. J. Smith, Adherence to antiretroviral HIV drugs: how many doses can you miss before resistance emerges, Proc. R. Soc. B., 273 (2006), 617-624. 1

[22] R. J. Smith, Explicitly accounting for antiretroviral drug uptake in theoretical HIV models predicts long-term failure of protease-only therapy, J. Theoret. Biol., 251 (2008), 227-237. 1 
[23] R. J. Smith, L. M. Wahl, Distinct effects of protease and reverse transcriptase inhibition in an immunological model of HIV-1 infection with impulsive drug effects, Bull. Math. Biol., 66 (2004), 1259-1283. 1, 2, 1

[24] R. J. Smith, L. M. Wahl, Drug resistance in an immunological model of HIV-1 infection with impulsive drug effects, Bull. Math. Biol., 67 (2005), 783-813. 1, 2, 1

[25] E. Tchetgen, E. H. Kaplan, G. H. Friedland, Public health consequences of screening patients for adherence to highly active antiretroviral therapy, JAIDS, 26 (2001), 119-129. 1

[26] P. Van den Driessche, J. Watmough, Reproductive numbers and subthreshold endemic equilibria for compartmental models of disease transmission, Math. Biosci., 180 (2002), 29-48. 2.1

[27] L. M. Wahl, M. A. Nowak, Adherence and drug resistance: predictions for therapy outcome, Proc. R. Soc. London B., 267 (2000), 835-843. 1, 3, 4

[28] W. Wang, X.-Q. Zhao, Threshold dynamics for compartmental epidemic models in periodic environments, J. Dynam. Differential Equations, 20 (2008), 699-717. 2.1, 2.1

[29] Y. Xiao, A semi-stochastic model for HIV population Dynamics, Int. J. Biomath., 2 (2009), 391-404. 1

[30] Y. Yang, Y. Xiao, Threshold dynamics for an HIV model in periodic environments, J. Math. Anal. Appl., 361 (2010), 59-68. 1

[31] Y. Yang, Y. Xiao, The effects of population dispersal and pulse vaccination on disease control, Math. Comput. Modelling, 52 (2010), 1591-1604. 2.1

[32] F. Zhang, X.-Q. Zhao, A periodic epidemic model in a patchy environment, J. Math. Anal. Appl., 325 (2007), 496-516. 2.2, 2.2

[33] X.-Q. Zhao, Dynamical Systems in Population Biology, Springer-Verlag, New York, (2003). 2.2 